\title{
Polyarteritis nodosa and drug abuse: is there a connection?
}

\author{
Noah Samuels, Ovadia Shemesh, Amos M Yinnon, Drora Fisher, Abraham S Abraham
}

\begin{abstract}
Summary
Drug abuse has been controversially linked to polyarteritis nodosa. A 28-yearold man with a history of drug abuse with inhaled heroin presented with an enigmatic illness consisting of refractory fever, bilateral pleural effusions, migratory polyarthritis, and a leukaemoid reaction. An abdominal angiography confirmed the diagnosis of polyarteritis nodosa, and treatment with both prednisone and cyclophosphamide resulted in significant clinical and laboratory improvement.
\end{abstract}

Keywords: polyarteritis nodosa, drug abuse, inhaled heroin

In 1970, the first of a series of papers showing the coincidence of drug abuse and a necrotising angiitis indistinguishable from polyarteritis nodosa was published. ${ }^{1}$ Many drugs have since been implicated, but because drug addicts often use more than one substance at the same time, and because of the impurity of the preparations, it has been difficult to establish a definite link between polyarteritis nodosa and any one drug.

We describe a young patient with a history of drug abuse with inhaled heroin, who was diagnosed as suffering from polyarteritis nodosa. He denied any past or present abuse of any other narcotic. This common form of drug abuse may be aetiologically relevant in the necrotising angiitis of drug abuse.

\section{Case report}

A 28-year-old man with an eight-year history of drug abuse with inhaled heroin only, presented to our department with high fever, productive cough, back and chest pain, and diarrhoea. Eight months earlier he had been hospitalised elsewhere with high fever, severe leukocytosis, arthritis of the right knee, and transudative pericarditis. He was treated with indomethacin, with subsequent clinical and laboratory improvement.

His present physical examination revealed Internal Medicine B, Shaare Zedek Medical Center, PO Box 3235, Jerusalem, Israel 91031 N Samuels

O Shemesh

AM Yinnon

D Fisher

AS Abraham

Accepted 24 January 1996 neutrophils, $13 \%$ band forms, $10 \%$ meta/ myelocytes, and $1 \%$ eosinophils); and a protions over both lung bases and diffuse wheezing. Laboratory tests demonstrated a normocytic normochromic anaemia (haemoglobin $11.0 \mathrm{~g} / \mathrm{dl}$ ); a leukaemoid reaction, reaching $65.7 \times 10^{9} / 1$ white blood cells $(75 \%$ longed prothrombin time (international normalised ratio of 1.6). Liver and renal function tests were all within normal limits, and urinalysis revealed only one erythrocyte and 2-4 leukocytes per high-powered field. All bacterial cultures were negative.

Serological tests were negative for hepatitis B surface antigen, although positive for IgG against hepatitis $\mathbf{B}$ core antigen. Tests were also negative for human immunodeficiency virus, Epstein-Barr virus, cytomegalovirus, hepatitis A and antineutrophil cytoplasmic antibodies. A Mantoux test was positive, with a skin reaction of $15 \mathrm{~mm}$. Chest X-rays demonstrated infiltrates in both lungs with bilateral effusions, both resolving spontaneously, along with echocardiographic evidence of pulmonary hypertension. A transoesophageal echocardiogram was performed, with no vegetations observed.

The patient developed polyarthritis of both wrists, the left hip, and the right knee, with sterile transudative synovial fluid in the knee. $\mathrm{He}$ had a persistent sub-febrile temperature and fluctuating leukocytosis. The fever, leukaemoid reaction, and polyarthritis were refractory to broad-spectrum antibiotics, partially resolving after initiation of naproxyn therapy. A suspicion of polyarteritis nodosa was confirmed by abdominal angiography which demonstrated multiple microaneurysms in the renal arterial tree bilaterally (figure). After initiation of treatment with prednisone and cyclophosphamide, the patient felt much better, with a decrease in the fever and leukocytosis.

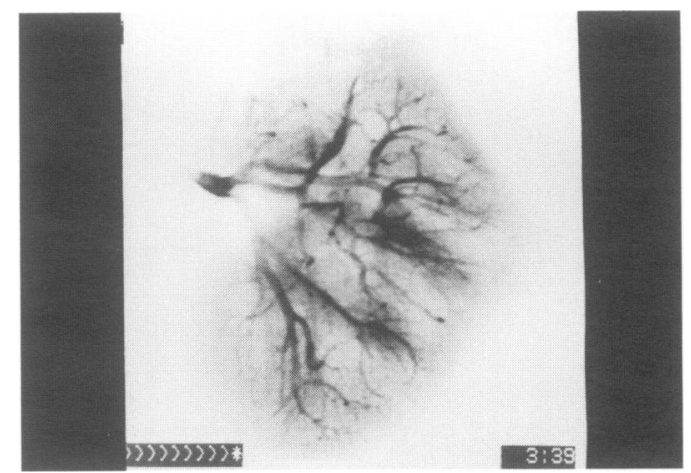

Figure 1 Renal arteriogram of the left kidney showing multiple aneurysms, the end result of the necrotising inflammatory process in the small and medium-sized arteries 


\section{Discussion}

Polyarteritis nodosa is a clinical and pathologic entity affecting small and medium-sized arteries. It is thought to be immune-mediated, with chronic panmural inflammatory lesions and multi-organ involvement. Without treatment, it carries a five-year survival rate of less than $15 \%$; with treatment survival is increased to as much as $50-60 \%{ }^{2}$

In 1970, Citron et al published the first paper linking drug abuse with necrotising angiitis indistinguishable from polyarteritis nodosa. ${ }^{1}$ Although the addicts studied had been using more than one drug at a time, the agent common to all appeared to be methamphetamine. Later reports, however, found cocaine, D-lysergic acid, ephedrine and intravenous heroin abuse in patients with necrotising angiitis, ${ }^{3,4}$ some of whom had cerebral vasculitis thought to be an early stage of polyarteritis nodosa, with serious complications such as stroke and haemorrhage. ${ }^{5}$

Many do not accept this link between narcotics and polyarteritis nodosa. One group examined 1031 drug addicts post-mortem and found no evidence of necrotising angiitis. ${ }^{6}$ Others acknowledge that polyarteritis nodosa is found more frequently among drug addicts, but explain this by the fact that the hepatitis B surface antigen, long associated with polyarteritis nodosa, ${ }^{2}$ is quite common among drug abusers. ${ }^{7}$ Still, between $70-90 \%$ of addicts with necrotising angiitis are $\mathrm{HBsAg}$ negative. ${ }^{8}$ Although our patient was also $\mathrm{HBsAg}$ negative, he did have evidence of previous infection with hepatitis $B$, as can be seen by the positive titre for IgG against hepatitis B core antigen. Other viruses have also been implicated (see box). ${ }^{2}$

Polyarteritis nodosa, with its multisystemic and often atypical presentation, is not an illness that is easily diagnosed. Certain information can increase the suspicion that a patient does

1 Citron P, Halpern M, McCarron M, et al. Necrotizing angiitis associated with drug abuse. $N$ Engl f Med 1970; 283 $1003-11$ 2 Conn DL. Polyarteritis. Rheum Dis Clin North Am 1990; 16:
341-62.

3 Kaye BR, Fainstat $M$. Cerebral vasculitis associated with cocaine abuse. $\mathscr{F} A M A$ 1987; 258: 2104-6.

4 King J, Richards $M$, Tress $B$. Cerebral arteritis associated with heroin abuse. Med 7 Aust 1978; 2: 444-5.
Risk factors associated with polyarteritis nodosa

Drugs

- methamphetamines

- cocaine (inhaled, intravenous)

- D-lysergic acid

- heroin (intravenous)

Viruses

- hepatitis B (HBsAg)

- human immunodeficiency virus

- cytomegalovirus

- human T-cell lymphotropic virus type I

- parvovirus

Box

indeed suffer from polyarteritis nodosa, such as a history of drug abuse or the presence of HBsAg. Early diagnosis and treatment are of utmost importance, and may significantly improve prognosis. Whether our patient's illness was related to his drug abuse or to his previous exposure to hepatitis $\mathbf{B}$ remains a mystery. Many of the clinical and laboratory presentations of his disease remain enigmatic, such as the pleural effusions and pulmonary hypertension which resolved spontaneously, the leukaemoid reaction, the prolonged prothrombin time (liver angiography was not performed), and the positive Mantoux reaction. Moreover, we have found no similar case linking inhaled heroin to polyarteritis nodosa. However, polyarteritis nodosa is a relatively uncommon disease, and there does appear to be a higher incidence among drug addicts. The fact that with an increase in the incidence of drug abuse this connection has not been shown more clearly may be attributed to a possible multifactorial aetiology in a rare disease. Further study may prove or disprove the connection.

5 Halpern M. Angiitis in drug abusers (letter). $N$ Engl $\mathcal{F}$ Med 1971; 284: 113 .

6 Baden $M$. Angiitis in drug abusers (letter). $N$ Engl $f$ Med 1971; 284: 111 .

7 Case records of the Massachusetts General Hospital: Case 36-1985. N Engl f Med 1985; 313: 622-31.

8 Citron P, Peters RL. Angiitis in drug abusers (letter). $N$ Engl f Med 1971; 284: 112 